\title{
Direct Mechanical Measurement of the Tensile Strength and Elastic Modulus of Multiwalled Carbon Nanotubes
}

\author{
B. G. Demczyk, Y. M. Wang, J. Cumings, M. Hetman, W. Han, A. Zettl and R. O. \\ Ritchie
}

University of California and Lawrence Berkeley National Laboratory, Berkeley, CA 94720

The discovery of fullerine-related carbon nanotube structures in 1991 [1] has renewed hopes of approaching the theoretical limit of tensile strength. Such structures grow as concentric cylinders and are nearly defect-free. Indirect measurements of the mechanical properties of these nanotubes have yielded very high moduli (Table 1). Direct tensile testing of individual tubes, however, has proved challenging, however, due to their extremely small size. We have developed a tensile testing stage for this purpose, using microfabrication techniques. This device enables the direct application of a tensile strain to individual nanotubes while they are viewed in a transmission electron microscope.

Carbon nanotubes were synthesized by arc discharge using a B-doped carbon anode[2]. The tensile testing device was fabricated from silicon utilizing microfabrication techniques. Tension was applied utilizing a piezoelectric manipulation holder, fabricated in-house, the details of which have been provided earlier [3-5].

Figures $1 \mathrm{a}$ and $1 \mathrm{~b}$ show the nanotube under test before and after tensile testing. Examination of individual video frames reveals a strain of just over $5 \%$, prior to failure. It should also be noted that no narrowing down of the nanotube was observed immediately before fracture, which is consistent with the lower temperature deformation mechanism predicted from dislocation theory [6]. Figure $1 \mathrm{~b}$ also reveals that the tube has apparently "healed" itself by forming a closed endcap. Based on our observed tensile force at failure (18 microNewtons) and the measured cross sectional area of the nanotube $\left(123 \mathrm{~nm}^{2}\right.$, based on a tube diameter of $\left.12.5 \mathrm{~nm}\right)$, our computed tensile stress to breakage is $0.15 \mathrm{TPa}$. This value generally is in general agreement with exceeds that predicted by the Orowan-Polyanyi relation, utilizing $\mathrm{E}=1.026 \mathrm{TPa}$ [7] for the graphene sheet. Although we were unable to obtain direct resolution of individual walls in the nanotube under test, both the "core" and "wall" regions are clearly visible. Based on measurements taken from these images, we calculate the number of walls as $\sim 13$. Our higher value of tensile stress to failure, coupled with the observation of the fracture surfaces suggests that the outermost walls break nearly at once. Examination of still micrographs before and after the event reveals much higher elongation, suggesting that the inner tubes may undergo a "sword in sheath" [8], (telescoping) phenomenon prior to failure.

The large elastic modulus and breaking strength determined for these multiwalled carbon nanotubes in addition to the elastic buckling observed during testing should render carbon nanotube reinforced composites particularly applicable where energy-absorbing properties are desired. 
Transmission electron microscopy was performed at the National Center for Electron Microscopy at the Lawrence Berkeley National Laboratory. Mechanical testing was supported in part by the Director of the Office of Science, Office of Science, Materials Sciences Division of the United States Department of Energy, contract number DEAC0376SF00098 and materials synthesis under grants DMR 98-01738 and DMR 95-01156 from the National Science Foundation.

\section{References}

[1] S. Iijima, Nature 354, 56 (1991).

[2] Ph. Redlich, J. Loeffler, P. M. Ajayan, J. Bill, F. Aldinger and M. Ruhle, Chem Phys. Lett. , 260, 465 (1996).

[3] J. Cumings and A. Zettl, Science 289, 602 (2000).

[4] J. Cumings, P. G. Collins and A. Zettl, Nature 406, 586 (2000).

[5] P. Poncharal, Z. L. Wang, D. Ugarte and W. A. DeHeer, Science 283, 1513 (1999).

[6] B. I. Yakobson, Appl. Phys. Lett. 72, 918 (1998).

[7] E. Orowan, Rep. Prog. Phys. 12, 185 (1949).

[8] M. F. Yu, B. S. Files, S. Arepalli and R. S. Ruoff, Phys. Rev. Lett. $\underline{84(24), 5552}$ (2000).

TABLE 1. Experimental mechanical properties of multiwalled carbon nanotubes

\begin{tabular}{lll}
\hline $\mathrm{E}(\mathrm{TPa})$ & $\sigma_{\mathrm{T}}(\mathrm{TPa})$ & Method \\
\hline $0.81(50 \%)$ & & AFM-2 ends clamped [34] \\
$1.28(40 \%)$ & & AFM- 1 end clamped [22] \\
$1.26(20 \%)$ & & TEM- thermally vibrating beam [23] \\
$0.1-1(\sim 1 / \mathrm{R})(30 \%)$ & & TEM-electrostatic deflection [15] \\
$0.27-0.95$ & $0.01-0.06$ & Dual AFM cantilevers [35] \\
$0.91(20 \%)$ & $0.15(30 \%)$ & TEM-direct tension [this work]
\end{tabular}

$\mathrm{E}=$ Young's Modulus, $\sigma_{\mathrm{T}}=$ tensile strength, $\mathrm{R}=$ nanotube radius, [ ]=uncertainty

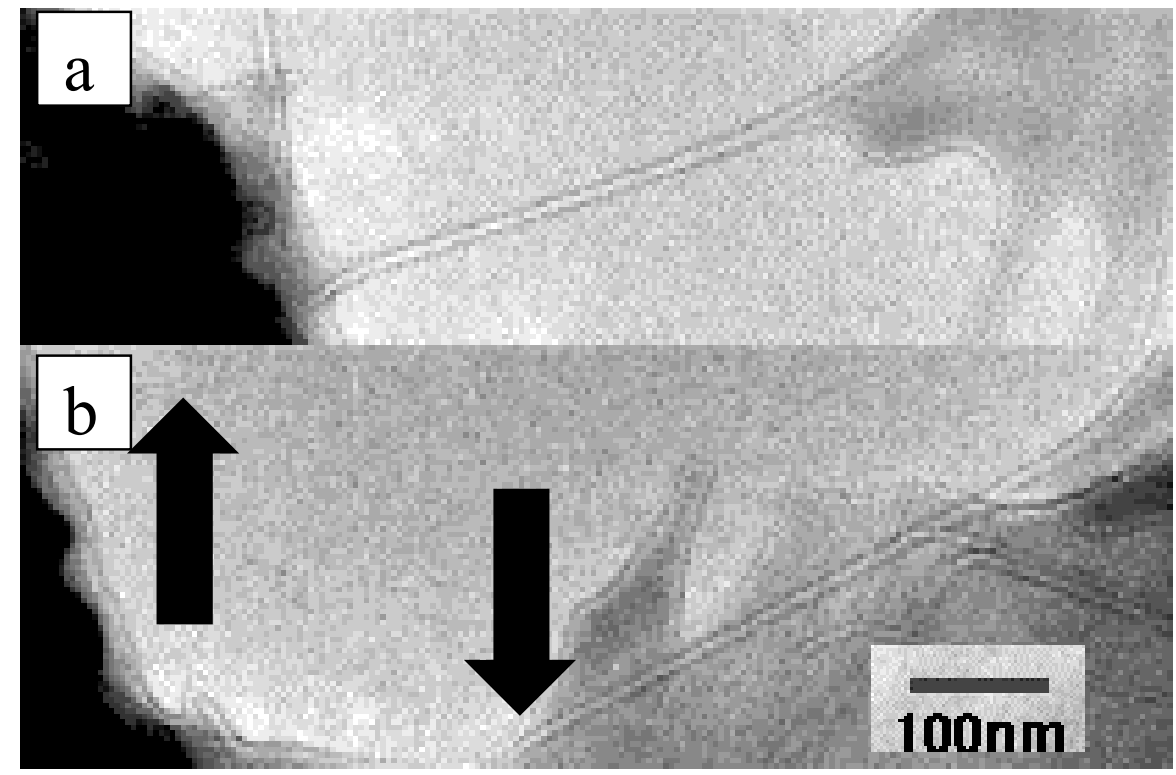

Fig. 1: Multiwall carbon nanotube: a) before, b) after breakage. 